\title{
Comparative Study on the Evaluation of Dental Bacterial Film Index Using Plaque Disclosing Agent and Quantitative Light Induced Fluorescence-Digital (QLF-D)
}

\author{
Cheon-Hee Lee ${ }^{1}$, Eun-Hee Lee ${ }^{2}$ \\ Department of Dental Hygiene, ${ }^{1}$ Andong Science College, Andong, ${ }^{2}$ Busan Women's College, Busan, Korea
}

Objective: The purpose of this study is to compare the dental biofilm index using quantitative light induced fluorescence-digital (QLF-D, All in One Bio Co., Seoul, Korea) with the dental biofilm test using a dye in the existing clinical setting and use it as a basis for improving the clinical utility and efficiency of dental biofilm management.

Methods: The correlation between total and anterior dental plaque index using dye and the QLF-D Simple Plaque Score (SPS) calculated using analysis software was analyzed for 63 adult males and females who agreed to participate in the study.

Results: There was a difference between the polymerase chain reaction (PCR) index using staining and the SPS index using QLF-D $(p<0.01)$, but as a result of the evaluation of the same site, it was confirmed that there was a positive correlation that the higher the PCR index, the higher the SPS index $(p<0.01)$.

Conclusion: Both the dental plaque index using QLF-D and the PCR index using the staining method are useful in evaluating the dental plaque index. This is considered presumed to be necessary.

Keywords: dental plaque, disclosing agent, quantitative light induced fluorescence-digital (QLF-D)

\section{Introduction}

Dental plaque is one of the main causes of periodontal disease, and if the dental plaque is not properly managed, anaerobic bacteria multiply and aggravate the inflammation of the gingiva [1]. Since the dental plaque is difficult to observe with the naked eye, various types of dental plaque indices have

Corresponding author Eun-Hee Lee

E-mail: arch1981@naver.com

(iD) https://orcid.org/0000-0003-1084-8286

Received December 1, 2021, Revised December 20, 2021, Accepted December 22, 2021 been developed to quantify the dental plaque deposited on the tooth surface. In general, the methods for examining dental plaque include plaque control record (polymerase chain reaction, PCR), Patient Hygiene Performance Index (PHPI), plaque free score, and Quigley Hein index [2]. This is because it is possible to obtain information about the patient's oral condition and brushing habits through the dental surface staining test, and it is one of the useful methods to motivate the oral hygiene management by maximizing the visual effect to the patient. However, since this test method has the characteristics of the examiner's subjective evaluation when evaluating the dental plaque, the reliability and accuracy are lowered. In addition, it takes a lot of time to color the tooth surface one by one and inspect the tooth after rinsing the mouth, it requires

Copyright (C) 2021. Korean Academy of Preventive Dentistry.

This is an Open Access article distributed under the terms of the Creative Commons Attribution Non-Commercial License (http://creativecommons.org/licenses/ by-nc/4.0) which permits unrestricted non-commercial use, distribution, and reproduction in any medium, provided the original work is properly cited. 
the effort of both the subject and the examiner, and it is difficult to cleanly remove the colored tooth surface after the examination [3]. On the other hand, quantitative light-induced fluorescence (QLF) is a device that detects red fluorescence generated from metabolites called porphyrin secreted by bacteria in the oral cavity, it is possible [4,5]. The quantity and quality of the deposited dental plaque can be evaluated on a scale of 0 to 5 using a dedicated analysis program (QA2 v 1.23; Inspektor research systems BV, The Netherlands). Various studies centered on the validity and reliability of dental plaque and dental examination through dental staining agent and quantitative light induced fluorescence-digital (QLF-D, All in One Bio Co., Seoul, Korea) and previous studies to prove the purpose of QLF were conducted [3,6-8]. As such, various studies are being actively conducted to quantify the dental plaque deposited on the tooth surface. In clinical dental hygiene education, evaluation of dental plaque is one of the key processes. Because it is very difficult for students in the dental hygiene department who are experiencing dental hygiene practice for the first time to accurately assess the level of dental plaque through an examination alone, repeated education and practice are conducted for all procedures performed by clinical dental hygienists. The dental hygienist identifies the patient's oral condition and establishes and carries out treatment and prevention and education plans for the patient [9], this is because they have a responsibility to educate themselves so that they can manage and prevent them [10]. Oh et al. [11] reported that students' dental condition evaluation competency was improved by repeating the experience of making correct judgments in a practical educational environment using the Q-ray view, which is the same principle as QLF-D. Although various studies on education and evaluation methods regarding the evaluation of the dental plaque are being actively conducted, the deposition pattern of the dental plaque appears in various forms depending on the shape and eruption state of the tooth. It is often not possible to fully reflect. In addition, there are not many studies proving the relationship between the amount of biofilm on the actual tooth surface and each index [12]. Therefore, this study compares the method using a colorant, one of the most skilled methods of evaluating dental plaque in the clinical dental hygiene process, and the dental plaque index using QLF, and intends to use it as basic data for efficient dental plaque evaluation.

\section{Materials and Methods}

\section{Subjects}

This study was conducted from March to June 2020 with 63 people who agreed to participate in the clinical dental hygiene course practice at a dental hygiene department located in Busan. The students who participated in the study received an education consisting of theory and practice on the imaging method and principle using QLF-D in advance and performed an examination together with an experienced investigator.

\section{Method}

\section{1) Dental plaque staining test (PCR)}

As the oral hygiene index measurement method, Sultan ${ }^{\circledR}$ (Sultan Chemists Corp., Englewood, NJ, USA), a tooth surface coloring agent, was used to measure all teeth in the oral cavity centered on the line angle. One point is given to each side, and the score is calculated by dividing it into the lowest 0 point and the highest 3 points. For comparison with the QLF-D imaging site, the color index of the maxillary 6 incisors and the color index of all teeth were recorded in the same area.

\section{2) Dental plaque test (SPS) using QLF-D}

For the dental plaque examination method using QLF-D, the subject was placed in the supine position on the unit chair in a dark room environment in the clinic, and 6 teeth of the maxillary anterior teeth were photographed from the front. After connecting the QLF-D to the laptop computer, the photographing program was executed and the photographed image of the subject was saved. The camera was positioned in front so that it could be seen from the gingiva to the cusp of the maxillary canine on the screen of Qraycam (All in One Bio Co., Seoul, Korea). The Simple Plaque Score (SPS), which evaluates the quantity and quality of dental plaque deposited using a dedicated analysis program (QA2 v 1.23; Inspektor research systems BV) through the images taken from QLF-D, is from 0 to 5. Scores were recorded for each site with a dot.

\section{Data analysis and statistical methods}

Measurement results and all collected data are presented in PASW Statistics for ver. 18 (IBM Co., Armonk, NY, USA). Frequency analysis and one-way ANOVA were performed to find out the general characteristics of the subjects and the average difference of the dental plaque index. In addition, Spearman Correlation Analysis was performed to investigate the correlation between evaluation indices according to the dental plaque measurement method.

\section{Results}

\section{General characteristics of subjects}

The general characteristics of the study subjects are as follows. The total number of subjects was 63 people, with 45 females 
(71.4\%) and 18 males (28.6\%). Ages of 20-29 were the most with 54 people (84.1\%) (Table 1).

\section{Comparison of dental plaque index according to measurement method}

The results of comparing the averages by dental plaque index were as follows (Table 2). The PCR index along the entire tooth surface and the maxillary 6 incisor PCR index were higher than the SPS index $(p<0.01)$. As a result of Scheffe's post hoc test, there was no average difference between the total dental plaque index using staining and the maxillary 6 anterior dental plaque index, but there was a difference between the SPS index using different measurement methods and the oral hygiene index using staining. Table 3 shows the results of correlation analysis to find out the correlation between each dental plaque indices. As the total PCR index and maxillary 6 incisor PCR index increased, it was confirmed that the SPS index also increased $(p<0.05$, $\mathrm{p}<0.01$ ). Also, it was found that there was a strong positive correlation between the PCR index of the maxillary 6 incisors using the staining method and the PCR for all teeth $(\mathrm{p}<0.01)$.

\section{Discussion}

Dental plaque is a thin bacterial film attached to the tooth surface and causes various diseases in the oral cavity [13]. Among them, periodontal disease is mainly caused by the inflammatory reaction caused by the dental plaque in the oral cavity [14] and is known to be related to systemic diseases [15]. These perio-

Table 1. General characteristics of the subjects

\begin{tabular}{ccc}
\hline Item & Characteristic & $\mathrm{N}(\%)$ \\
\hline Sex & Male & $18(28.6)$ \\
& Female & $45(71.4)$ \\
Age & $20-29$ & $54(84.1)$ \\
& $30-39$ & $3(4.8)$ \\
& $40-49$ & $2(3.2)$ \\
& $>66$ & $4(6.3)$ \\
Total & & $63(100.0)$ \\
\hline
\end{tabular}

Table 2. Comparison of dental plaque index by measurement method

\begin{tabular}{lccccc}
\hline \multicolumn{1}{c}{ Item } & $\mathrm{N}$ & $\mathrm{M} \pm \mathrm{SD}$ & $\mathrm{F}$ & $\mathrm{p}$-value & Scheffe $^{* *}$ \\
\hline PCR A & 63 & $52.68 \pm 22.80$ & 149.72 & $0.00^{*}$ & $\mathrm{a}, \mathrm{b}>\mathrm{C}$ \\
PCR B & 63 & $59.22 \pm 27.79$ & & & \\
SPS & 63 & $0.81 \pm 1.13$ & & & \\
\hline
\end{tabular}

PCR A for all teeth. PCR B modified PCR for anterior labial. $M_{ \pm} S D$ : mean \pm standard deviation, PCR: polymerase chain reaction, SPS: Simple Plaque Score. ${ }^{*} \mathrm{p}<0.01 .{ }^{*}$ a: PCR A, b: PCR B, c: SPS. dontal diseases and various oral diseases can be prevented through dental plaque management. Therefore, the most basic thing to prevent oral diseases and maintain a healthy oral condition is to manage the dental plaque. In order to smoothly manage the dental plaque, in the dental hygiene process, an appropriate prevention and education plan is established by identifying the oral condition of the subject, and education and practice are being operated to perform it [16]. Therefore, for the objective evaluation of oral conditions, dental plaque examination is essential. To date, various studies have been conducted to quantitatively evaluate the stained dental plaque on the tooth surface [12], but overcoming the disadvantages of time and cost of consumables and reduced reliability due to the fatigue of the examiner. To this end, in the field of dental hygiene, the use of QLF-D is gradually increasing in student education and subject management [17-19]. In this study, the correlation between the dental plaque index measured using QLF-D and the pigmented dental plaque index was evaluated. As a result of the study, as in the previous study $[3,4]$ that analyzed the correlation between the red fluorescence of the dental plaque photographed with QLF-D and the dental plaque using a colorant, a high level of correlation was found in the SPS index and the maxillary 6 incisor PCR index $(r=0.323, p<0.01)$. However, in this study, when measuring the dental plaque index using QLF-D, only the maxillary 6 incisors are compared with the pigmentation index. In the case of the PCR index, only the presence or absence of the colored bacterial film is evaluated, so the qualitative characteristics of the dental plaque are reflected. Therefore, there is a limitation in evaluating the total dental plaque index in the oral cavity. As shown in Figure 1, it was found that when the pictures taken with QLF-D and coloring were performed, the tooth surface coloring agent evaluated more areas. The SPS, which is the criterion for judging the dental plaque index, scores higher when the maturity of the dental plaque is high, and in the case of PCR that calculates the stained area and area, even if the maturity is relatively high, the PCR index increases when the stained area is widened. It is thought that the average difference between the pigmented dental plaque index and the

Table 3. Correlation between dental plaque index by measurement site and method

\begin{tabular}{cccc}
\hline Item & PCR A $(\mathrm{n}=64)$ & PCR B $(\mathrm{n}=64)$ & SPS \\
\hline PCR A & 1 & & \\
PCR B & $0.771^{* *}$ & 1 & \\
SPS & $0.300^{*}$ & $0.323^{* *}$ & 1 \\
\hline
\end{tabular}

PCR A for all teeth. PCR B modified PCR for anterior labial. PCR: polymerase chain reaction, SPS: Simple Plaque Score. ${ }^{*} p<0.05$. $* * \mathrm{p}<0.01$. 

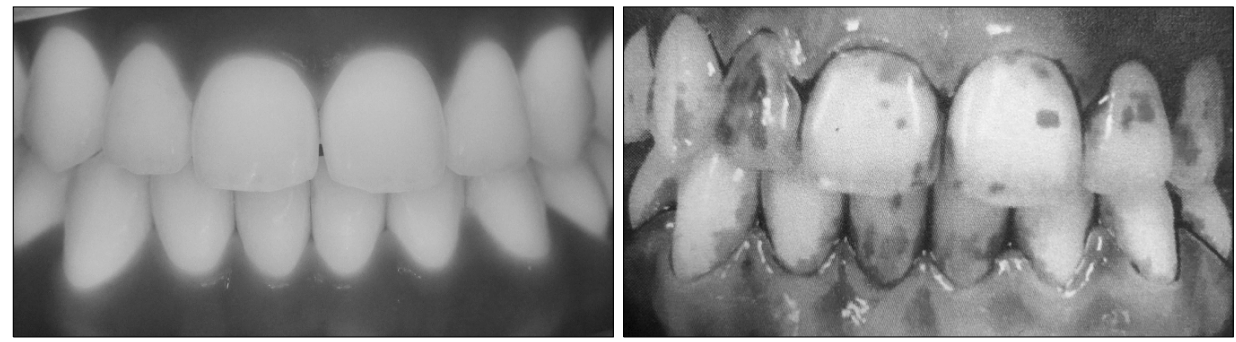

Figure 1. Comparison of dental plaque taken with QLF-D and dental plaque on stained area. QLF-D: quantitative light induced fluorescencedigital.

SPS index is due to the difference in the discrimination criteria according to the amount of dental plaque and the maturity of the dental plaque. However, it showed a trend similar to the results of previous studies that evaluated the mature dental plaque on all teeth, and it is considered that there is a difference in the criteria for evaluating the dental plaque as a comparative study of the existing colored dental plaque index and the measurement index through image analysis.

Starting with this study, it is considered that continuous development of an analysis program useful for monitoring periodontal status is required along with the development of an index that reflects the qualitative characteristics of the dental plaque well compared to the existing evaluation method when inspecting the dental plaque.

\section{Conclusion}

This study was conducted to compare the dental plaque index using a colorant and QLF-D to provide an accurate diagnosis for dental plaque evaluation and to use it as basic data. As a result of the analysis, the following conclusions were drawn.

1. As a result of comparing the average dental plaque index, there was no difference between the total PCR index and the maxillary 6 incisor PCR index, but the SPS index and the total PCR index and the maxillary 6 incisor PCR index using staining were statistically significant $(\mathrm{p}<0.01)$.

2. As a result of the correlation analysis, positive correlation was also found between the overall PCR index and the SPS index $(\mathrm{p}<0.05)$, and the maxillary 6 incisor PCR index and the SPS index $(p<0.01)$. Also, the PCR and 6 incisor PCR indexes for all teeth showed a positive correlation $(p<0.01)$.

Through the above results, a statistically significant result was found in the correlation analysis between the dental plaque indices using the dental staining agent and QLF-D, whereas the difference in the average was somewhat due to the maturation of the dental plaque compared to the index proportional to the area where the dental plaque was colored. It is thought to have appeared due to the difference in the indices being evaluated. The comparative study between the method using the dental staining agent and the QLF-D, an objective tool, is meaningful in that the dental hygienist presented the basis for the evaluation of the dental plaque to efficiently perform the dental plaque management task. In the future, it is expected that various studies that can be quantified more efficiently in the evaluation of dental plaque in clinical practice requiring accuracy will be conducted.

\section{Conflict of Interest}

No potential conflict of interest relevant to this article was reported.

\section{ORCID}

Cheon-Hee Lee, https://orcid.org/0000-0002-3203-8025

Eun-Hee Lee, https://orcid.org/0000-0003-1084-8286

\section{References}

1. Socransky SS, Haffajee AD. The bacterial etiology of destructive periodontal disease: current concepts. J Periodontol 1992;63(4 Suppl):322-31.

2. O'Leary TJ, Drake RB, Naylor JE. The plaque control record. J Periodontol 1972;43:38.

3. Kim M, Lee SY, Cho YS. Validity and reliability of the plaque score using Qraycam. J Dent Hyg Sci 2015;15:377-82.

4. Hwang HR, Cho YS, Kim BI. Assessment of clinical applicability of a new plaque scoring system using quantitative light-induced fluorescence-digital. J Dent Hyg Sci 2014;14:150-7.

5. Kim BI. QLF concept and clinical implementation. J Korean Dent Assoc 2011;49:443-50.

6. Kang HK. The effect of oral health education using Qraycam. J Korea Converg Soc 2019;10:333-40.

7. Min JH, Yoon HC, Kim JK, Kang SM, Kim BI. Assessment of acidogenic potential for dental biofilms by periodontal health condition. J Dent Hyg Sci 2015; 15:202-8.

8. Lee ES, Jong EDJ, Jung HI, Kim BI. Red fluorescence of dental biofilm as an indicator for assessing the efficacy of antimicrobials. J Biomed Opt 2018;23:015003.

9. Wilkins EM. Clinical practice of the dental hygienist. 10th ed. Philadelphia: Wolters Kluwer/Lippincott Williams \& Wilkins; 2009:101-11.

10. Barnes CM. Dental hygiene participation in managing incipient 
and hidden caries. Dent Clin North Am 2005;49:795-813.

11. Oh HY, Jung HI, Ku HM, Kim BI. The effects of the Q-ray view on reliability of assessing a tooth status for dental hygiene process. J Dent Hyg Sci 2014;14:461-7.

12. Kim JS, Yang YH, Jun EJ, Kim JB, Jeong SH. Comparison of traditional dental plaque indices with real stained plaque area. J Korean Acad Oral Health 2017;41:262-6.

13. Fejerskov O, Nyvad B, Kidd EAM. Dental caries: what is it? In: Fejerskov O, Nyvad B, Kidd EAM. Dental caries: the disease and its clinical management. 3rd ed. Oxford: Wiley/Blackwell; 2015:7-10.

14. Jo YS, Lee CJ, Choi YH, Na EJ, Park JH. A clinical study on the ultra-sonic stimulation for gingival disease patient. Int J Clin Prev Dent 2013;9:39-45.
15. Heo SY, Lee EH. The correlation among systemic health, hypertension and oral health of Korean adults. Int J Clin Prev Dent 2018;14:112-8.

16. Han SY, Kim NH, Yoo JH, Kim CS, Chung WG. Current status of clinical dental hygiene education based on dental hygiene process of care. J Dent Hyg Sci 2009;9:271-8.

17. Kim HE. Quantitative light-induced fluorescence: a potential tool for dental hygiene process. J Dent Hyg Sci 2013;13:115-24.

18. Lee SR, Kim JH, Huh SY. Comparative study of dental plaque reduction according to various mouthwashes using quantitative light induced fluorescence-digital. J Dent Hyg Sci 2013;13:434-9.

19. Lee SY, Lim SR, Bae HS. Applicability evaluation of quantitative light-induced fluorescence-digital and Cariview in cries prediction study. J Dent Hyg Sci 2013;13:403-9. 